\title{
On wave turbulence in MHD
}

\author{
S. Galtier ${ }^{1,2}$, S. V. Nazarenko ${ }^{1}$, and A. C. Newell ${ }^{1}$ \\ ${ }^{1}$ Mathematics Institute, University of Warwick, Coventry, CV4 7AL, UK \\ ${ }^{2}$ Present address: Institut d'Astrophysique Spatiale, Bâtiment 121, Université Paris-Sud, F-91405 Orsay Cedex, France
}

Received: 18 September 2000 - Revised: 18 December 2000 - Accepted: 22 January 2001

\begin{abstract}
We describe the fundamental differences between weak (wave) turbulence in incompressible and weakly compressible MHD at the level of three-wave interactions. The main difference is in the structure of the resonant manifolds and the mechanisms of redistribution of spectral densities along the applied magnetic field $\boldsymbol{B}_{\mathbf{0}}$. Similar to pure acoustic waves, a three-wave resonance between collinear wavevectors is observed but, in addition, we also have a resonance through tilted planes and spheres. The properties of resonances and their consequences for the asymptotics are also discussed.
\end{abstract}

\section{Introduction}

It is widely recognised that magnetohydrodynamics (MHD) provides a first satisfactory description of many turbulent plasmas encountered in astrophysics (Priest, 1982; Grappin et al., 1983; Heiles et al., 1993; Pouquet, 1993; Marsch and Tu, 1994; Parker, 1994; Tu and Marsch, 1995; Goldstein et al., 1997), as well as in laboratory devices, such as tokamaks (Taylor, 1986; Gekelman and Pfister, 1988). MHD turbulence differs significantly from hydrodynamic turbulence in the fact that a strong magnetic field has a non-trivial effect on the dynamics. It was Iroshnikov (1963) and Kraichnan (1965) (hereafter IK) who first recognised that the presence of Alfvén waves travelling in opposite directions along local large magnetic fields leads to the weakening of energy transfer to small scales and, therefore, to a modification of the scaling of the (Kolmogorov) energy spectrum from $k^{-5 / 3}$, for neutral fluids, to $k^{-3 / 2}$.

In the IK phenomenology, MHD turbulence is assumed to be $3 \mathrm{D}$ isotropic. However, in many realistic situations, the presence of strong magnetic fields is observed, which makes MHD turbulence strongly anisotropic. Anisotropy is manifested in a two-dimensionalisation of the turbulence spec-

Correspondence to: S. Galtier

(sebastien.galtier@ias.fr) trum in a plane transverse to the locally dominant magnetic field and in inhibiting spectral energy transfer along the direction parallel to the field (Montgomery and Turner, 1981; Montgomery and Matthaeus, 1995; Matthaeus et al., 1996; Kinney and McWilliams, 1998). Replacing the 3D isotropy assumption by a $2 \mathrm{D}$ one, and retaining the rest of the IK dimensional analysis, gives a $k_{\perp}^{-2}$ spectrum $\left(\boldsymbol{B}_{\mathbf{0}}=B_{0} \hat{\boldsymbol{e}}_{\|}\right.$, the applied magnetic field, $k_{\|} \stackrel{\perp}{=} \cdot \hat{\boldsymbol{e}}_{\|}, \boldsymbol{k}_{\perp}=\boldsymbol{k}-k_{\|} \hat{\boldsymbol{e}}_{\|}$, $\left.k_{\perp}=\left|\boldsymbol{k}_{\perp}\right|\right)$.

MHD turbulence in the presence of a strong uniform magnetic field has been recently investigated by several authors. $\mathrm{Ng}$ and Bhattacharjee $(1996,1997)$; Bhattacharjee and $\mathrm{Ng}$ (2000) developed a theory of weakly interacting Alfvén wave packets which takes into account anisotropy. This leads to certain predictions for the turbulence spectra based on some additional phenomenological assumptions. In Webb et al. (2000), weakly nonlinear three-wave resonant interactions in 1D compressible MHD are studied in the presence of entropy waves and in the case where waves have a welldefined phase. More recently, Galtier et al. (2000) developed a rigorous theory of weak Alfvén turbulence in incompressible MHD. The derivation of such a theory, at the level of three-wave interactions, via a systematic asymptotic expansion in powers of small nonlinearity leads to some exact results which will be discussed in detail in the next section. One of the main results obtained is that the $k_{\perp}^{-2}$ spectrum is an exact finite flux Kolmogorov solution of the wave turbulence equation and that it corresponds to local turbulence: the collision integral converges on this spectrum. This property is a necessary and sufficient condition to apply the Zakharov transformation which reveals the $k_{\perp}^{-2}$ solution. Convergence means that the interactions between triads of waves with large wavevector separations decay sufficiently fast for the collision integral to exist and, therefore, the main contribution comes from triads with similar wavevectors. On the other hand, according to the IK picture, the existence of the (short) Alfvén waves themselves in MHD turbulence is due to the action of a strong magnetic field carried by the large scales. This is obviously another type of non-locality which 
is assumed here and which is not going to be examined.

Observations of turbulence in the solar wind and the interstellar medium reveal the presence of density variations (Armstrong et al., 1981; Bavassano et al., 1982; Grappin et al., 1990; Marsch and Tu, 1990a,b, 1993). Therefore, any theory based on incompressible MHD has to be seen as a first attempt to describe such turbulent plasmas. However, it is a (much) more difficult task to include compressibility effects in a rigorous theory like weak turbulence. Here, we would like to emphasise that weak turbulence has to be seen as a useful theoretical framework to understand media like the solar wind or the interstellar medium. It cannot be seen stricto sensu as a model for such media, since we observe only a moderate anisotropy (moderate background magnetic field). However, this asymptotic model (limit of strong background magnetic field), by revealing properties which are quite different to the isotropic case (strong turbulence), tells us what direction the dynamics must point towards when the anisotropy becomes stronger and thus, it gives important physical information about the medium itself. For example, several observations reveal spectra with a power-law index between -1.9 and -2 (Burlaga and Goldstein, 1984; Burlaga et al., 1987; Spangler and Gwinn, 1990), which is definitively steeper than the (Kolmogorov or Iroshnikov-Kraichnan) phenomenological predictions for isotropic turbulence. As it is stated in Bhattacharjee and $\mathrm{Ng}$ (2000), such a steepening of the spectrum can be due to shocks and discontinuities, but it could also be due to turbulence.

In Sect. 3, we present the basis on which a weakly compressible turbulence theory can be derived. In addition to Alfvén waves, fast and slow magneto-acoustic waves must now to be taken into account. The resonant manifolds are much richer than in the incompressible case and they allow transfer along the strong uniform magnetic field. The way in which this transfer is possible is similar to the one for acoustic waves, namely along rays in all directions, but also through tilted planes and spheres. The two last mechanisms of transfer are due to the interaction between magnetic and acoustic waves. We also show that the presence of double resonances may lead to different asymptotics according to the dimension of the problem.

\section{Weak Alfvénic turbulence}

There is an extensive literature devoted to weak turbulence (see e.g. Vedenov, 1967; Benney and Newell, 1969; Sagdeev and Galeev, 1969; Zakharov, 1974; Akhiezer et al., 1975; McIvor, 1977; Zakharov et al., 1992). Basically, the essence of weak turbulence is the statistical study of large ensembles of weakly interacting waves via a systematic asymptotic expansion in powers of small nonlinearity. This approach leads finally to the derivation of "kinetic equations" for quantities like the wave energy. Previously, it was believed that this asymptotic expansion was consistent only for dispersive or semi-dispersive (e.g. acoustic) waves. The study by Galtier et al. (2000) shows that non-dispersive Alfvén waves are, in fact, a unique exception to this rule which gives very particular properties to weak Alfvénic turbulence. The physical reason is that for non-dispersive waves, all wavepackets propagate with the same group velocity even if their wavenumbers are different. Thus, no matter how weak the nonlinearity is, the energy exchanged between the wavepackets will be accumulated over a long time and it may not be considered small, as it would be required in the weak turbulence theory. Alfvén waves are an exception because the nonlinear interaction coefficient for co-propagating waves is null, whereas the counter-propagating wavepackets pass through each other in a finite time and exchange only small amounts of energy over the waveperiod timescale which makes the weak turbulence approach applicable in this case.

\subsection{Kinetic equation for the energy spectrum}

In this section, we would like to briefly review the kinetic equation for the energy spectrum. Details of the derivation are given in Galtier et al. (2000), but for the coherence of this paper, some important steps of the derivation will be outlined.

We start with the 3D incompressible MHD equations for the velocity $\boldsymbol{v}$ and the Alfvén velocity $\boldsymbol{b}$

$$
\begin{aligned}
& \left(\partial_{t}+\boldsymbol{v} \cdot \nabla\right) \boldsymbol{v}=-\nabla\left(P / \rho_{0}+\boldsymbol{b}^{2} / 2\right)+\boldsymbol{b} \cdot \nabla \boldsymbol{b}+v \nabla^{2} \boldsymbol{v}, \\
& \left(\partial_{t}+\boldsymbol{v} \cdot \nabla\right) \boldsymbol{b}=\boldsymbol{b} \cdot \nabla \boldsymbol{v}+\eta \nabla^{2} \boldsymbol{b} \\
& \nabla \cdot \boldsymbol{v}=0 \\
& \nabla \cdot \boldsymbol{b}=0
\end{aligned}
$$

where $P$ is the thermal pressure, $\boldsymbol{b}=\boldsymbol{B} / \sqrt{\mu_{0} \rho_{0}}, \rho_{0}$ the uniform density, $\mu_{0}$ the magnetic permeability, $v$ the viscosity and $\eta$ the magnetic diffusivity. We follow the eulerian wave turbulence approach which can be found, for instance, in Benney and Newell (1969). We assume the presence of a strong uniform magnetic field $B_{0}$ and that the wave amplitudes are small, which allow us to introduce a small parameter $\epsilon \ll 1$. Note that this approach does not contain any constraints which might reflect polarization preferences. Introducing potentials $\psi^{s}$ and $\phi^{s}$ which are related to the Elsässer fields (whose amplitude are proportional to $\epsilon$ ) $z^{s}=\boldsymbol{v}+s \boldsymbol{b}$ $(s= \pm 1)$ via

$z^{s}=\nabla \times\left(\psi^{s} \hat{\boldsymbol{e}}_{\|}+\nabla \times \phi^{s} \hat{\boldsymbol{e}}_{\|}\right)$,

we derive (Galtier et al., 2000) a closed system of kinetic equations for the second correlations of the Fourier transforms $\hat{\psi}^{s}(\boldsymbol{k})$ and $\hat{\phi}^{s}(\boldsymbol{k})$ in the inviscid case. The physically important combinations of these correlators are the total energy $e^{s}(\boldsymbol{k})$, poloidal and toroidal energies, $\Phi^{s}(\boldsymbol{k})$ and $\Psi^{s}(\boldsymbol{k})$, pseudo magnetic $I^{S}(\boldsymbol{k})$ and magnetic $R^{s}(\boldsymbol{k})$ helicities whose definitions are

$$
\begin{aligned}
& \left\langle\hat{\psi}^{s}(\boldsymbol{k}) \hat{\psi}^{s}\left(\boldsymbol{k}^{\prime}\right)\right\rangle=\delta\left(\boldsymbol{k}+\boldsymbol{k}^{\prime}\right) \Psi^{s}\left(\boldsymbol{k}^{\prime}\right), \\
& \left\langle\hat{\phi}^{s}(\boldsymbol{k}) \hat{\phi}^{s}\left(\boldsymbol{k}^{\prime}\right)\right\rangle=\delta\left(\boldsymbol{k}+\boldsymbol{k}^{\prime}\right) \Phi^{s}\left(\boldsymbol{k}^{\prime}\right), \\
& \left\langle\hat{\psi}^{s}(\boldsymbol{k}) \hat{\phi}^{s}\left(\boldsymbol{k}^{\prime}\right)\right\rangle+\left\langle\hat{\phi}^{s}(\boldsymbol{k}) \hat{\psi}^{s}\left(\boldsymbol{k}^{\prime}\right)\right\rangle=\delta\left(\boldsymbol{k}+\boldsymbol{k}^{\prime}\right) R^{s}(\boldsymbol{k}), \\
& \left\langle\hat{\psi}^{s}(\boldsymbol{k}) \hat{\phi}^{s}\left(\boldsymbol{k}^{\prime}\right)\right\rangle-\left\langle\hat{\phi}^{s}(\boldsymbol{k}) \hat{\psi}^{s}\left(\boldsymbol{k}^{\prime}\right)\right\rangle=\delta\left(\boldsymbol{k}+\boldsymbol{k}^{\prime}\right) I^{s}(\boldsymbol{k}),
\end{aligned}
$$


$e^{s}(\boldsymbol{k})=\boldsymbol{k}_{\perp}^{2}\left(\Psi^{s}(\boldsymbol{k})+k^{2} \Phi^{S}(\boldsymbol{k})\right)$,

where \langle\rangle denotes an ensemble average. Because of the assumption of spatial homogeneity, it is equivalent to think of it as an average over the base coordinate.

The resulting kinetic equations are lengthy (Galtier et al., 2000 ) and for what follows, it suffices to write only the equation for the energy spectrum $e^{s}(\boldsymbol{k})$,

$$
\begin{aligned}
& \frac{\partial e^{s}(\boldsymbol{k})}{\partial t}=\frac{\pi \varepsilon^{2}}{b_{0}} \int\left[\left(L_{\perp}^{2}-\frac{X^{2}}{k^{2}}\right) \Psi^{s}(\boldsymbol{L})\right. \\
& -\left(k_{\perp}^{2}-\frac{X^{2}}{L^{2}}\right) \Psi^{s}(\boldsymbol{k})+\left(L_{\perp}^{2} L^{2}-\frac{k_{\|}^{2} W^{2}}{k^{2}}\right) \Phi^{s}(\boldsymbol{L}) \\
& -\left(k_{\perp}^{2} k^{2}-\frac{k_{\|}^{2} Y^{2}}{L^{2}}\right) \Phi^{s}(\boldsymbol{k})+\left(\frac{k_{\|} X Y}{L^{2}}\right) I^{s}(\boldsymbol{k}) \\
& \left.-\left(\frac{k_{\|} X W}{k^{2}}\right) I^{s}(\boldsymbol{L})\right] Q_{k}^{-s}(\boldsymbol{\kappa}) \delta\left(\kappa_{\|}\right) \delta_{\boldsymbol{k}, \boldsymbol{\kappa} \boldsymbol{L}} d \boldsymbol{\kappa} d \boldsymbol{L},
\end{aligned}
$$

with

$$
\begin{aligned}
& \delta_{\boldsymbol{k}, \kappa \boldsymbol{L}}=\delta(\boldsymbol{L}+\boldsymbol{\kappa}-\boldsymbol{k}), \\
& Q_{k}^{-s}(\boldsymbol{\kappa})=X^{2} \Psi^{-s}(\boldsymbol{\kappa})+X\left(k_{\|} \kappa_{\perp}^{2}-\kappa_{\|} Y\right) I^{-s}(\boldsymbol{\kappa}) \\
& +\left(\kappa_{\|} Y-k_{\|} \kappa_{\perp}^{2}\right)^{2} \phi^{-s}(\kappa), \\
& X=k_{\perp} \kappa_{\perp} \sin \theta, \\
& Y=k_{\perp} \kappa_{\perp} \cos \theta \text {, } \\
& W=k_{\perp}^{2}-L_{\perp}^{2}-k_{\perp} \kappa_{\perp} \cos \theta,
\end{aligned}
$$

where $\theta$ is the angle between $\boldsymbol{k}_{\perp}$ and $\boldsymbol{\kappa}_{\perp}$, and with

$d \kappa_{\perp}=L_{\perp} d \kappa_{\perp} d L_{\perp} / k_{\perp} \sin \theta$,

$\cos \theta=\left(\kappa_{\perp}^{2}+k_{\perp}^{2}-L_{\perp}^{2}\right) / 2 \kappa_{\perp} k_{\perp}$.

The integral on the RHS of (11) (called the collision integral or CI) is generally non-zero. It is an equation for the slow time change of the leading term $e^{s}(\boldsymbol{k})$ in an asymptotic expansion for the energy density and is chosen so as to remove all secular terms $\left(\epsilon^{2} t\right)^{N}, N=1,2,3, \ldots$, which appear due to the three-wave processes. Higher order corrections to the kinetic equations can arise from higher order processes, such as four-wave interactions, but they do not affect the result on the time scale $t_{t r}=\sqrt{\mu_{0} \rho_{0}} / \epsilon^{2} B_{0} k_{\perp}$. Even at longer time scales, these corrections are only significant if they cause a redistribution of energy in $\boldsymbol{k}$ space not mediated by three-wave processes. The main point, however, is that on the time scale $t_{t r}$, three-wave interactions dominate. This subtle point is at the origin of a recent controversy (Sridhar and Goldreich, 1994; Goldreich and Sridhar, 1995). The original error of Goldreich and Shridhar (GS) was in failing to recognise that the power (the Fourier transform of the two-point correlation) contained in a zero mean signal need not be zero, even at zero wavenumber (frequency). A longer discussion is given in Nazarenko et al. (2000).

\subsection{General properties}

The delta function in the CI arises because of the three-wave frequency resonance condition. Thus, in any resonantly interacting wave triad $(\boldsymbol{k}, \boldsymbol{\kappa}, \boldsymbol{L})$, there is always one wave that corresponds to a purely 2D motion, having no dependence on the direction parallel to the uniform magnetic field, whereas the other two waves have equal parallel components of their corresponding wavenumbers, viz. $L_{\|}=k_{\|}$. Such a property was recently discussed in Kinney and McWilliams (1998). This means that the parallel components of the wavenumber enter in the kinetic equation of the total energy $e^{s}(\boldsymbol{k})$ as an external parameter and that the dynamics is decoupled at each level of $k_{\|}$. In other words, there is no energy transfer associated with the three-wave resonant interaction along the $k_{\|}$-direction in $\boldsymbol{k}$-space for the total energy: the resonant manifolds of the energy redistribution process foliate $\boldsymbol{k}$-space. The absence of the parallel transfer was also discussed in Kinney and McWilliams (1998) who also presented a numerical confirmation of this fact. Furthermore, for every triad of Alfvén waves $\boldsymbol{k}, \boldsymbol{L}$ and $\boldsymbol{\kappa}$ (such that $\kappa_{\|}=0$ ), the energy is conserved within two co-propagating waves having wavevectors $\boldsymbol{k}$ and $\boldsymbol{L}$, due to the symmetry of CI with respect to changing $\boldsymbol{k} \leftrightarrow \boldsymbol{L}$ and $\boldsymbol{\kappa} \leftrightarrow-\boldsymbol{\kappa}$.

The first consequence of the fact that there is no transfer of the total energy in the $k_{\|}$-direction in $\boldsymbol{k}$-space is an asymptotic two-dimensionalisation of the energy spectrum $e^{s}(\boldsymbol{k})$. Namely, the 3D initial spectrum spreads over the transverse wavenumbers, $\boldsymbol{k}_{\perp}$, but remains of the same size in the $k_{\|}$ direction, and the support of the spectrum becomes very flat (pancake-like) for a large amount of time. The two-dimensionalisation of weak MHD turbulence has been observed in laboratory experiments (Robinson and Rusbridge, 1971), and in the the solar wind data (Belcher and Davis, 1971; Bavassano et al., 1982; Horbury et al., 1995; Bieber et al., 1996), as well as in many direct numerical simulations of the threedimensional MHD equations (Oughton et al., 1994; Kinney and McWilliams, 1998). From the mathematical point of view, the two-dimensionalisation of the total energy means that, for a large amount of time, the energy spectrum $e^{s}(\boldsymbol{k})$ is supported on a volume of wavenumbers such that for most of them $k_{\perp} \gg k_{\|}$. This implies that $\Psi^{s}(\boldsymbol{k})$ and $\Phi^{s}(\boldsymbol{k})$ are also supported on the same anisotropic region of wavenumbers because both of them are non-negative. This fact allows one to expand the integrands in the kinetic equations in powers of small $k_{\|} / k_{\perp}$. At leading order (and taking $\Phi^{s}(\boldsymbol{k})=0$ ), one obtains

$$
\begin{aligned}
& \frac{\partial\left[k_{\perp}^{2} \Psi^{s}(\boldsymbol{k})\right]}{\partial t}=\frac{\pi \varepsilon^{2}}{b_{0}} \int\left[\left(L_{\perp}^{2}-\frac{X^{2}}{k_{\perp}^{2}}\right) \Psi^{s}(\boldsymbol{L})\right. \\
& \left.-\left(k_{\perp}^{2}-\frac{X^{2}}{L_{\perp}^{2}}\right) \Psi^{s}(\boldsymbol{k})\right] X^{2} \Psi^{-s}(\boldsymbol{\kappa}) \delta\left(\kappa_{\|}\right) \delta_{\boldsymbol{k}, \boldsymbol{\kappa} \boldsymbol{L}} d \boldsymbol{\kappa} d \boldsymbol{L} .
\end{aligned}
$$

An important consequence of the dynamical decoupling at different $k_{\|}$values within the kinetic equation formalism is that the set of purely 2D modes (corresponding to $k_{\|}=0$ ) evolves independently from the 3D part of the spectrum (with 
$\left.k_{\|} \neq 0\right)$ and can be studied separately. This property was also observed in direct numerical simulations (Kinney and McWilliams, 1998).

It is important to note that the kinetic equations themselves are applicable to a description of $k_{\|}=0$ modes only if the correlations of the dynamical fields decay in all directions, so that their spectra are sufficiently smooth for all wavenumbers, including the ones with $k_{\|}=0$. To be precise, the characteristic scale $k_{\|}$over which the spectra can experience significant changes must be greater than $\epsilon^{2}$ because the kinetic equation arises from a limit process $B_{0} k_{\|} t \rightarrow \infty, \epsilon^{2} B_{0} k_{\perp} t$ finite, where $k_{\|}$and $k_{\perp}$ are dominant parallel and perpendicular wavenumbers. The parallel wavenumber $k_{\|}$enters equation (13) only as an external parameter. In other words, the wavenumber space is foliated into the dynamically decoupled planes and $k_{\|}=$constant. Thus, the large-time asymptotic solution can be found in the following form,

$\Psi^{S}\left(\boldsymbol{k}_{\perp}, k_{\|}\right)=f\left(k_{\|}\right) \Psi^{S}\left(\boldsymbol{k}_{\perp}, 0\right)$,

where $f$ is some arbitrary function of $k_{\|}$satisfying the conditions $f(0)=1$. Substituting this formula into (13), one can readily see that the solution of the $3 \mathrm{D}$ equations is reduced to solving a 2D problem for $\Psi^{s}\left(\boldsymbol{k}_{\perp}, 0\right)$.

Using the Zakharov transform, we solved this 2D problem and found the exact stationary solutions in power laws which are

$E_{\perp}^{s}\left(k_{\perp}, 0\right)=k_{\perp}^{3} \Psi^{s}\left(k_{\perp}, 0\right) \sim k_{\perp}^{n_{s}}$,

where $n_{+}+n_{-}=-4$. In the particular case of a zero correlation between the velocity and the magnetic field, one has $n_{+}=n_{-}=-2$. The Kolmogorov constants appearing in front of the spectra are computed exactly and found to depend on the amount of correlation between the velocity and the magnetic field. We also prove that the collision integral converges for all $-3<n_{s}<-1$, which gives an a posteriori justification for the application of the Zakharov transform. Numerical simulations show the $k_{\perp}^{-2}$ solution to be attractive and that the small scales are reached in a finite time, i.e. in a catastrophic way. In particular, and very much to our surprise, it was observed (Galtier et al., 2000) that the spectral energy density propagates to large wavenumbers following a stationary $k_{\perp}^{-7 / 3}$-spectrum and not a $k_{\perp}^{-2}$-spectrum; the latter obtained during the self-similar decay phase. When the dissipative scale is reached, the $k_{\perp}^{-7 / 3}$ solution suddenly turns into the finite energy flux spectrum $k_{\perp}^{-2}$ with a change of the slope propagating from small scales to large scales. This picture is very different from the scenario proposed by Falkovich and Shafarenko (1991) for finite capacity spectra and has many of the hallmarks of a dramatic new type of phase transition. Note that a similar behaviour is also observed in the formation of Bose condensates (Svistunov, 1991; Lacaze et al., 1999).

\section{Compressible MHD}

We would like now to emphasise the important effect of weak compressibility in weak MHD turbulence. Compressible MHD turbulence has been the subject of many recent theoretical (see e.g. Marsch and Mangeney, 1987; Tu, 1987; Marsch and Tu, 1989; Zhou and Matthaeus, 1989; Zank and Matthaeus, 1993; Bhattacharjee et al., 1998) and numerical (see e.g. Vázquez-Semadeni et al., 1995; Matthaeus et al., 1996; Roberts et al., 1996; Ghosh and Goldstein, 1997) works. For example, a turbulence description for an inhomogeneous solar wind has led to the development of two-scale dynamical theories in terms of correlation functions where different plasma $\beta$ (the ratio of thermal to magnetic pressure) regimes have been considered (Marsch and Mangeney, 1987; Zank and Matthaeus, 1993). Elsässer variables appear to be very convenient in incompressible MHD, comparable to the context of weak turbulence (Galtier et al., 2000). Their generalisation to compressible MHD including density variation has been obtained by Marsch and Mangeney (1987). However, canonical variables (see below) seem to be more appropriate than compressive Elsässer variables in building a weak compressible turbulence theory.

Our aim here is not to derive a complete weak MHD turbulence theory (the detailed analysis is being carried out by us in a separate work; see, however, Kaburaki and Uchida (1971) for a discussion about weak nonlinear coupling of MHD wave modes), but rather to show by considering the resonant manifolds in the limit of small compressibility that the physical picture of spectral densities redistribution may differ significantly from the incompressible case. The main difference is that transfer along the strong uniform magnetic field is no longer forbidden; the way by which this transfer is allowed is similar to acoustic waves, namely along rays in all directions (Newell and Aucoin, 1971; L'vov et al., 1997), but in addition, we have transfer through tilted planes and spheres. The two last mechanisms find their origin in the interaction between magnetic and acoustic waves.

\subsection{MHD waves}

We consider now the 3D compressible inviscid MHD equations

$$
\begin{aligned}
& \partial_{t} \rho+\nabla(\rho \boldsymbol{v})=0, \\
& \partial_{t} \boldsymbol{v}+\boldsymbol{v} \cdot \nabla \boldsymbol{v}=-\frac{\nabla P}{\rho}+\frac{1}{\mu_{0} \rho}(\nabla \times \boldsymbol{B}) \times \boldsymbol{B}, \\
& \partial_{t} \boldsymbol{B}=-\nabla \times(\boldsymbol{B} \times \boldsymbol{v}), \\
& P=P_{0}\left(\frac{\rho}{\rho_{0}}\right)^{\gamma}, \\
& \nabla \cdot \boldsymbol{B}=0,
\end{aligned}
$$

where $\rho$ is the density and $\gamma$ the polytropic index. Note that since we consider polytropic gas (19), we do not have any entropy waves such as those found in the works of Webb et al. (1999, 2000). We consider waves with small amplitude in the presence of a strong uniform magnetic field $B_{0}$ and we 
introduce the following notations

$\boldsymbol{B}=B_{0} \hat{\boldsymbol{e}}_{\|}+\epsilon B_{0} \boldsymbol{b}_{\mathbf{1}}$,

$\boldsymbol{v}=\epsilon C_{A} \boldsymbol{v}_{\mathbf{1}}$,

$\rho=\rho_{0}+\epsilon \rho_{0} \rho_{1}$,

where $\epsilon \ll 1, C_{A} \equiv B_{0} / \sqrt{\mu_{0} \rho_{0}}$ is the Alfvén speed and $\hat{\boldsymbol{e}}_{\|}=(0,0,1)$. Let us use a $2.5 \mathrm{D}$ geometry to derive the well-known dispersion relations. MHD equations linearised and expressed in terms of the dimensionless variables (21)(23) give in the Fourier space (the symbol` stands for the Fourier transform)

$\partial_{t} \hat{\boldsymbol{W}}=-i C_{A} k\left(\begin{array}{cccccc}0 & M_{A} \sin \theta & 0 & M_{A} \cos \theta & 0 & 0 \\ M_{A} \sin \theta & 0 & 0 & 0 & -1 & 0 \\ 0 & 0 & 0 & 0 & 0 & -\cos \theta \\ M_{A} \cos \theta & 0 & 0 & 0 & 0 & 0 \\ 0 & -1 & 0 & 0 & 0 & 0 \\ 0 & 0 & -\cos \theta & 0 & 0 & 0\end{array}\right) \hat{\boldsymbol{W}}$,

where $\hat{\boldsymbol{W}} \equiv\left(M_{A} \hat{\rho}_{1}, \hat{v}_{1 x}, \hat{v}_{1 y}, \hat{v}_{1 z}, \hat{b}_{1 x} / \cos \theta, \hat{b}_{1 y}\right), M_{A} \equiv$ $C_{S} / C_{A}, C_{S} \equiv \sqrt{\gamma P_{0} / \rho_{0}}$ is the sound speed, $\boldsymbol{k}=$ $k(\sin \theta, 0, \cos \theta), \theta=\left(\widehat{\boldsymbol{k}, \boldsymbol{B}_{\mathbf{0}}}\right)$ and $k=|\boldsymbol{k}|$. Note that in (24) the divergence free condition for the magnetic field is used to reduce the initial system of seven variables to six variables. With this choice of variables, the expression for total energy of the system per unit volume $E_{T}$ (the sum of kinetic, magnetic and internal energies) is then simply written as

$\left\langle E_{T}\right\rangle=\frac{B_{0}^{2}}{2 \mu_{0}}+\frac{P_{0}}{\gamma-1}+\frac{1}{2} \epsilon^{2} \rho_{0} C_{A}^{2} \sum_{\ell=1}^{6}\left\langle W_{\ell}^{2}\right\rangle$,

where $W_{\ell}$ is the $\ell$-th component of $\boldsymbol{W}$. The diagonalisation of the matrix leads finally to the well-known dispersion relations (see e.g. Jeffrey and Taniuti (1964))

$\partial_{t} A_{j}^{s}(\boldsymbol{k})+i \omega_{j}^{s} A_{j}^{s}(\boldsymbol{k})=0$,

$$
\begin{aligned}
\omega_{j}^{s} & =s \omega_{j}(\boldsymbol{k})=s k C_{A} M_{j} \quad(j=1,2,3), \\
M_{1} & \equiv M_{F} \\
& =\left(1+M_{A}^{2}+\sqrt{\frac{\left(1+M_{A}^{2}\right)^{2}-4 M_{A}^{2} \cos ^{2} \theta}{2}}\right)^{1 / 2}, \\
M_{2} & \equiv M_{S} \\
& =\left(1+M_{A}^{2}-\sqrt{\frac{\left(1+M_{A}^{2}\right)^{2}-4 M_{A}^{2} \cos ^{2} \theta}{2}}\right)^{1 / 2}, \\
M_{3} & =\cos \theta,
\end{aligned}
$$

where $M_{F / S}$ and $M_{A}$ are, respectively, the phase velocity of fast/slow magneto-acoustic wave and Alfvén wave; $s= \pm$ refers to the direction of propagation of waves; the normal vector is

$\boldsymbol{A}^{s}(\boldsymbol{k})=\left(\begin{array}{c}\frac{-1}{2\left(M_{F}^{2}-M_{S}^{2}\right)}\left\{M_{A} \sin \theta\left(\hat{W}_{1}+s M_{S} \hat{W}_{4}\right)+\left(M_{S}^{2}-1\right)\left(\hat{W}_{5}-s M_{F} \hat{W}_{2}\right)\right\} \\ \frac{1}{2\left(M_{F}^{2}-M_{S}^{2}\right)}\left\{M_{A} \sin \theta\left(\hat{W}_{1}+s M_{F} \hat{W}_{4}\right)+\left(M_{F}^{2}-1\right)\left(\hat{W}_{5}-s M_{S} \hat{W}_{2}\right)\right\} \\ \frac{1}{2}\left\{\hat{W}_{6}-s \hat{W}_{3}\right\}\end{array}\right)$.

Then, after having inverted the previous system to express $\hat{W}$ in terms of the normal vector, we can write the expressions for the invariants of compressible MHD whose density spectra are for total energy (at order $\epsilon^{2}$ )

$$
\begin{aligned}
E_{T}(\boldsymbol{k}) & =\rho_{0} C_{A}^{2}\left\{2\left(1-M_{F}^{2}\right) q_{11}^{+-}(\boldsymbol{k})\right. \\
& \left.+2\left(1-M_{S}^{2}\right) q_{22}^{+-}(\boldsymbol{k})+q_{33}^{++}(\boldsymbol{k})+q_{33}^{--}(\boldsymbol{k})\right\},
\end{aligned}
$$

and for the cross-correlation (whose definition is $E_{C}=$ $\frac{1}{2}\langle\boldsymbol{v} \cdot \boldsymbol{B}\rangle$ in the physical space)

$E_{C}(\boldsymbol{k})=\frac{1}{2}\left(q_{33}^{--}(\boldsymbol{k})-q_{33}^{++}(\boldsymbol{k})\right)$, where the energy tensor is defined as

$$
\begin{aligned}
q_{j_{1} j_{2}}^{s_{1} s_{2}}(\boldsymbol{k}) \delta\left(\boldsymbol{k}+\boldsymbol{k}^{\prime}\right) \equiv & \left\langle A_{j_{1}}^{s_{1}}(\boldsymbol{k}) A_{j_{2}}^{s_{2}}\left(\boldsymbol{k}^{\prime}\right)\right\rangle e^{-i\left(\omega_{j_{1}}^{s_{1}}+\omega_{j_{2}}^{s_{2}}\right) t} \\
& =\left\langle a_{j_{1}}^{s_{1}}(\boldsymbol{k}) a_{j_{2}}^{s_{2}}\left(\boldsymbol{k}^{\prime}\right)\right\rangle .
\end{aligned}
$$

The last invariant, the magnetic helicity $H_{M}$, is null in the weak turbulence limit, since it involves terms of type $q_{3}^{s_{1} s_{2}}$ with $j=1,2$.

The normal vector is the starting point to derive in the interacting representation the fundamental equation for the wave amplitude, where the slow variation in time is due to weak nonlinearities. Formally, this equation may be written as 


$$
\begin{aligned}
\partial_{t} a_{j}^{s}(\boldsymbol{k}, t)= & i \epsilon \int C_{m, n, s_{1}, s_{2}}^{j, s}(\boldsymbol{k}, \boldsymbol{\kappa}, \boldsymbol{L}) a_{m}^{s_{1}}(\boldsymbol{\kappa}) a_{n}^{s_{2}}(\boldsymbol{L}) \\
& \cdot e^{i\left(s \omega_{j}(\boldsymbol{k})-s_{1} \omega_{m}(\boldsymbol{\kappa})-s_{2} \omega_{n}(\boldsymbol{L})\right) t} \delta_{\boldsymbol{k}, \boldsymbol{\kappa} \boldsymbol{L}} d_{\boldsymbol{\kappa} \boldsymbol{L}},
\end{aligned}
$$

where the rule of summation over repeated indices is assumed (i.e. for $m, n, s_{1}$ and $s_{2}$ ). The interacting coefficient $C_{m, n, s_{1}, s_{2}}^{j, s}(\boldsymbol{k}, \boldsymbol{\kappa}, \boldsymbol{L})$, which contains information on nonlinearities, is generally much more complicated than in the incompressible case. Zero interacting coefficients are observed for

$$
\begin{aligned}
& C_{m, n, s_{1}, s_{2}}^{j, s}=0 \text { for } j=1,2 \text { and }\left\{\begin{array}{l}
m=3 ; n=1,2 \\
\text { or } \\
m=1,2 ; n=3
\end{array}\right. \\
& C_{m, n, s_{1}, s_{2}}^{j, s}=0 \text { for } j=3 \text { and }\left\{\begin{array}{l}
m=n \\
\text { or } \\
n, m=1,2
\end{array}\right.
\end{aligned}
$$

In particular, it means that for this choice of geometry if Alfvén waves are initially absent, they cannot be produced by nonlinearities (Nakariakov et al., 1997). The exponentially oscillating term in (35) plays a fundamental role in the closure procedure: its exponent should not vanish when $\boldsymbol{k}=\boldsymbol{\kappa}+\boldsymbol{L}$. We shall look now for the resonant manifold's solution of the system

$s \omega_{j}(\boldsymbol{k})=s_{1} \omega_{m}(\boldsymbol{\kappa})+s_{2} \omega_{n}(\boldsymbol{L})$,

$\boldsymbol{k}=\boldsymbol{\kappa}+\boldsymbol{L}$.

But, in the general case, it is not an easy task to solve this system; therefore, in the next section, we will restrict our attention to the weakly compressible limit.

\subsection{Weakly compressible limit in 3D}

We want to determine the resonant manifolds for three-wave interactions in the 3D case in the weakly compressible limit. As it was explained before in incompressible MHD, the resonant manifolds foliate wavevector space, which leads to an absence of energy transfer along the main magnetic field. We will see that this transfer is no longer forbidden when magneto-acoustic waves are taken into account.

The starting point of our study is the function

$h(\boldsymbol{\kappa} ; \boldsymbol{k})=s_{1} \omega_{m}(\boldsymbol{\kappa})+s_{2} \omega_{n}(\boldsymbol{k}-\boldsymbol{\kappa})-s \omega_{j}(\boldsymbol{k})$.

The resonant manifold $M$ is then defined as the ensemble of $\boldsymbol{\kappa}\left(s, s_{1}, s_{2}\right.$ and $\boldsymbol{k}$ given $)$ for which $h(\boldsymbol{\kappa} ; \boldsymbol{k})=0$. As it will be explained later, we are also interested to know if these resonances are double, i.e. if in the Taylor expansion of $h$ in the neighbourhood of $M$

$$
\begin{gathered}
h(\boldsymbol{\kappa} ; \boldsymbol{k})=h\left(\boldsymbol{\kappa}^{(0)} ; \boldsymbol{k}\right)+\left(\boldsymbol{\kappa}-\boldsymbol{\kappa}^{(0)}\right) \cdot \nabla_{\kappa} h\left(\boldsymbol{\kappa}^{(0)} ; \boldsymbol{k}\right)+\ldots \\
\left(\boldsymbol{\kappa}^{(0)} \in M\right)
\end{gathered}
$$

the gradient is zero or not. Indeed the presence of double resonances may change the asymptotic according to the dimension of the problem. This is true, for example, for acoustic waves (Newell and Aucoin, 1971; L'vov et al., 1997).
In the weakly compressible limit $\left(M_{A} \rightarrow+\infty, C_{A}\right.$ finite $)$ we have

$\omega_{F}(\boldsymbol{k})=k C_{A}\left(M_{A}+\frac{\sin ^{2} \theta}{2} \frac{1}{M_{A}}\right)+O\left(\frac{1}{M_{A}^{3}}\right)$,

$\omega_{S}(\boldsymbol{k})=k_{\|} C_{A}+O\left(\frac{1}{M_{A}^{2}}\right)$,

$\omega_{A}(\boldsymbol{k})=k_{\|} C_{A}$,

which means that slow magneto-acoustic waves degenerate with the Alfvén waves. At the main order the gradient of $h$ reads (only the projection on $\boldsymbol{n}$, vector normal to the resonant manifold $M$, is considered since the tangential part is automatically null)

$$
\begin{array}{r}
\nabla_{\kappa} h \cdot \boldsymbol{n}=-\left[\mathcal{F}_{F / S}(\boldsymbol{\kappa})+\mathcal{F}_{F / S}(\boldsymbol{k}-\boldsymbol{\kappa})\right] \boldsymbol{\kappa}^{2} \\
+\mathcal{F}_{F / S}(\boldsymbol{k}-\boldsymbol{\kappa}) \boldsymbol{k} \cdot \boldsymbol{\kappa}-s_{2} \omega_{F / S}(\boldsymbol{k}-\boldsymbol{\kappa}) \\
\cdot \frac{\left(k_{\|} \kappa_{\perp}-k_{\perp} \kappa_{\|}\right)}{(\boldsymbol{k}-\boldsymbol{\kappa})^{2}}
\end{array}
$$

with

$\mathcal{F}_{F / S}(\boldsymbol{k})= \pm s \frac{C_{A}^{2}}{\omega_{F / S}(\boldsymbol{k})} \frac{k_{\perp} k_{\|}}{k^{2}}$,

where a positive sign must be taken for $F$ and a negative sign for $S$. In particular, we note the useful property

$\mathcal{F}_{F / S}(a \boldsymbol{k})=\mathcal{F}_{F / S}(\boldsymbol{k}) /|a|$

For Alfvén waves, we note that

$\nabla_{\kappa} \omega_{A}(\boldsymbol{\kappa})=-\nabla_{\kappa} \omega_{A}(\boldsymbol{k}-\boldsymbol{\kappa})=C_{A} \hat{\boldsymbol{e}}_{\|}$.

We are now going to consider the different possibilities for three-wave interactions.

\subsubsection{FFF interaction}

For interaction between three fast magneto-acoustic waves $(\mathrm{FFF})$, we have to solve at the main order

$h(\boldsymbol{\kappa} ; \boldsymbol{k})=C_{S}\left(s_{1} \kappa+s_{2}|\boldsymbol{k}-\boldsymbol{\kappa}|-s k\right)=0$.

If we do not consider the trivial case $s=-s_{1}=-s_{2}$ for which the solution is made by three null vectors, the resonances always occur between wave vectors purely collinear. More precisely, if we define $\boldsymbol{\kappa}=\alpha \boldsymbol{k}$, we have

$\alpha \leq 0 \quad$ if $s=-s_{1}=s_{2}$,

$0 \leq \alpha \leq 1 \quad$ if $s=s_{1}=s_{2}$,

$1 \leq \alpha \quad$ if $s=s_{1}=-s_{2}$.

The representation of the resonant manifolds in Fourier-space is given in Fig. 1. We observe the same situation as for pure acoustic waves: the resonant manifolds correspond to rays in all directions. From (45) and (46) we see that we have, in fact, double resonances where both $h$ and $\nabla_{\boldsymbol{k}} h$ vanish on the resonant manifold. 


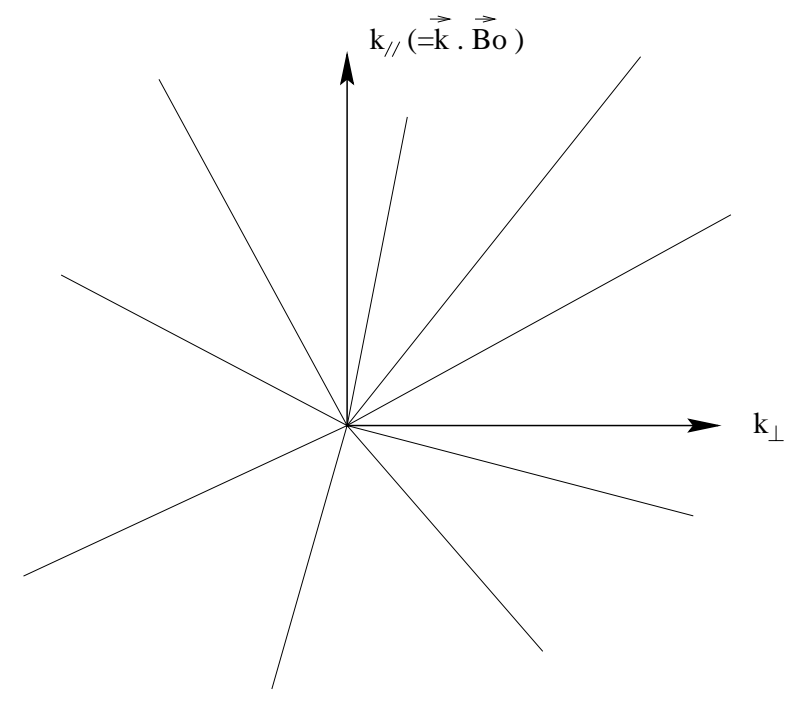

Rays

Fig. 1. A representation of the resonant manifolds in Fourier-space for FFF interaction. In the weakly compressible limit it is defined as the ensemble of $\boldsymbol{\kappa}$ for which $h(\boldsymbol{\kappa} ; \boldsymbol{k})=C_{S}\left(s_{1} \kappa+s_{2}|\boldsymbol{k}-\boldsymbol{\kappa}|-s k\right)=$ 0 . Similar to acoustic turbulence the resonant manifold is composed of rays.

\subsubsection{SSS/SSA/SAA/AAA interaction}

Interaction between three slow magneto-acoustic waves (SSS) gives

$h(\boldsymbol{\kappa} ; \boldsymbol{k})=C_{A}\left(\left(s_{1}-s_{2}\right) \kappa_{\|}-\left(s-s_{2}\right) k_{\|}\right)=0$.

For case $s=s_{1}=s_{2}$, the relation is always satisfied which means that the closure procedure does not work. However, this conclusion depends, of course, on the value of the interacting coefficient. This point will be discussed in Sect. 3.3. For $s=s_{1}=-s_{2}$, the resonant manifold is the plane $\kappa_{\|}=k_{\|}$; for $s=-s_{1}=s_{2}$, it is the plane $\kappa_{\|}=0$; and for $-s=s_{1}=s_{2}$, it is the plane $k_{\|}=0$ (so for $\kappa_{\|}=0$ ). Double resonances are observed for cases $s_{1}=s_{2}$. For interactions of type SSA, SAA and AAA, the resonance conditions are the same and so are the results. Note that we already know for incompressible MHD (AAA) that interacting coefficients are non-zero only for $s_{1}=-s_{2}$. A representation of the resonant manifolds in Fourier-space is given in Fig. 2. It shows planes perpendicular to the background magnetic field direction: the resonant manifolds foliate wavevector space.

\subsubsection{FFS/FFA interaction}

Interaction between two fast and one slow magneto-acoustic waves (FFS) leads to (if the S-wave is supported on the wavevector $\boldsymbol{k}$ )

$h(\boldsymbol{\kappa} ; \boldsymbol{k})=C_{S}\left(s_{1} \kappa+s_{2}|k-\kappa|\right)=0$.

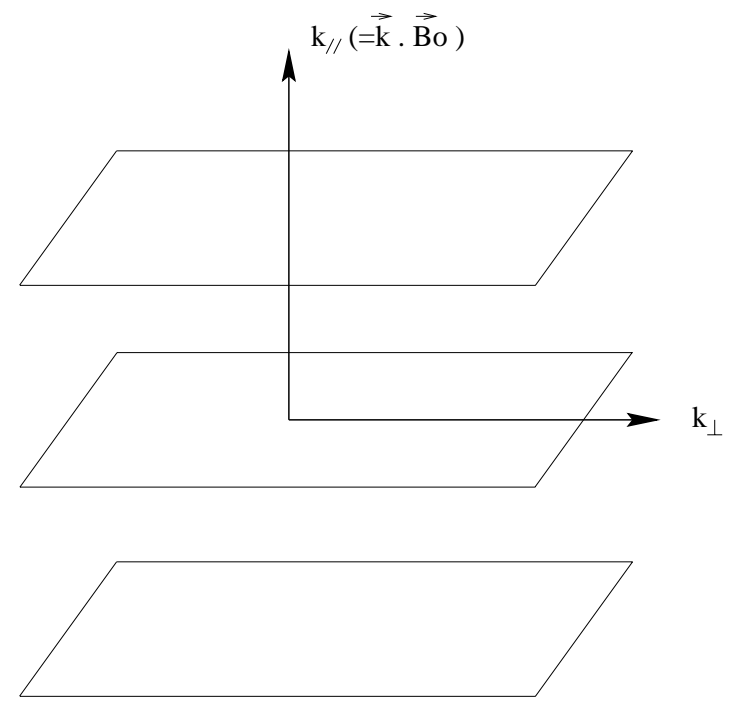

Planes

Fig. 2. A representation of the resonant manifolds in Fourierspace for SSS/SSA/SAA/AAA interaction. In the weakly compressible limit it is defined as the ensemble of $\boldsymbol{\kappa}$ for which $h(\boldsymbol{\kappa} ; \boldsymbol{k})=$ $C_{A}\left(\left(s_{1}-s_{2}\right) \kappa_{\|}-\left(s-s_{2}\right) k_{\|}\right)=0$. Similar to Alfvénic turbulence (AAA) the resonant manifolds foliate $\boldsymbol{k}$ space: they are composed of planes perpendicular to the background magnetic field direction.

When $s_{1}=s_{2}$, the trivial solution is $\kappa=0$; otherwise, the resonant manifold is defined by $\kappa=|k-\kappa|$, which defines a (tilted) plane intersecting $\boldsymbol{k}$ at the middle of its length. If the $\mathrm{S}$-wave is supported on the wavevector $\boldsymbol{k}-\boldsymbol{\kappa}$, the nontrivial solution for the resonant manifolds is $\kappa=k$, which defines a sphere of radius $k$. Finally, if the $S$-wave is supported on the wavevector $\boldsymbol{\kappa}$, then the resonant manifold is just $\kappa=0$. The resonances are generally simple except if vectors are collinear. Interaction between two fast magneto-acoustic waves and one Alfvén wave (FFA) leads to the same type of results. A representation of non-trivial resonant manifolds in Fourier-space is given in Fig. 3: it shows tilted planes and spheres.

\subsubsection{FSS/FSA/FAA interaction}

For interaction between one fast and two slow magnetoacoustic waves (FSS), the resonant manifold is readily obtained: in the weakly compressible limit, the wavevector which supports the F-wave has to be zero. Then the resonant manifold is defined by $\boldsymbol{\kappa}=0$, or $\boldsymbol{\kappa}=\boldsymbol{k}$ (if we do not consider the irrelevant case $\boldsymbol{k}=0$ ). We only have simple resonances. Interaction of type FSA and FAA gives the same kind of results. Since the resonant manifolds are trivial, a geometrical representation will not be given. 


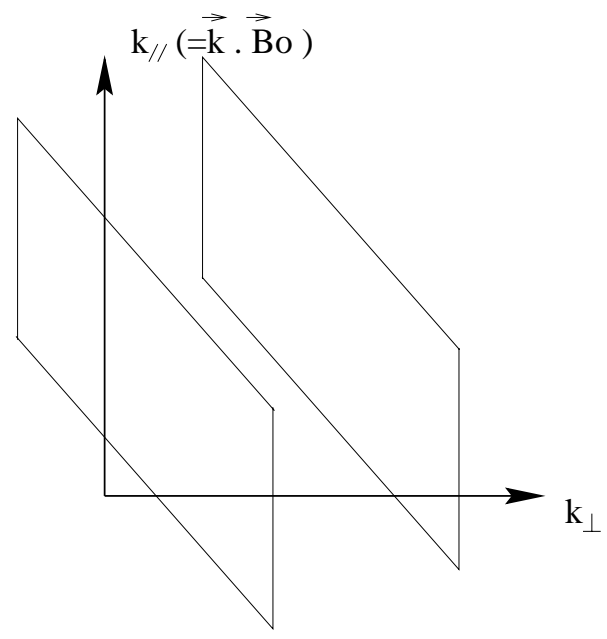

Tilted planes

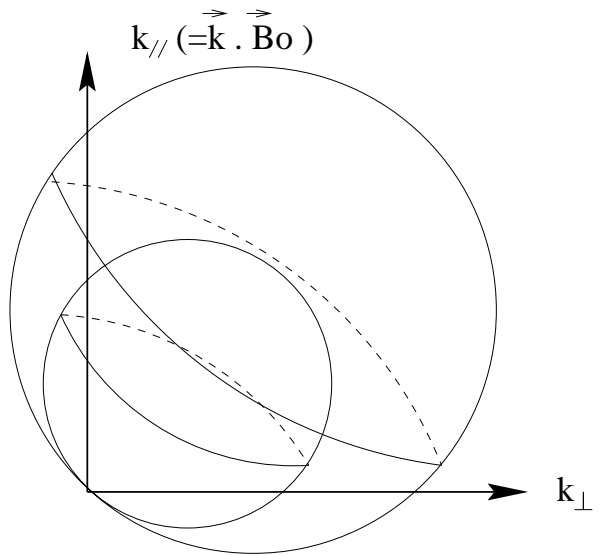

Spheres

Fig. 3. A representation of the resonant manifolds in Fourier-space for FFS/FFA interaction. In the weakly compressible limit it is defined as the ensemble of $\boldsymbol{\kappa}$ for which $h(\boldsymbol{\kappa} ; \boldsymbol{k})=C_{S}\left(s_{1} \kappa+s_{2}|k-\kappa|\right)=0$. The non-trivial resonant manifold is composed of tilted planes and spheres.

\subsection{General discussion}

The resonant manifolds in weakly compressible MHD are richer than for the incompressible case. A representation of the resonant manifolds in Fourier-space is given in Figs. 1-3. By revealing the geometry of the resonant manifolds it gives us an idea of how the spectral densities can redistribute along the mean magnetic field direction $\boldsymbol{B}_{\mathbf{0}}$. Indeed, the weak turbulence formalism leads to the derivation of kinetic equations in Fourier-space for the redistribution of spectrum densities (such as energy spectrum) via resonant exchange between the three waves $\boldsymbol{k}, \boldsymbol{\kappa}$ and $\boldsymbol{L}$ lying on the manifold $M$. Therefore, we see that an energy redistribution along the $\boldsymbol{B}_{\mathbf{0}}$ direction may appear in the weak compressible MHD turbulence regime: it may appear first along rays, i.e. by a resonance between collinear wave vectors, secondly along spheres, and thirdly along tilted planes. The first mechanism of redistribution is similar to what we know for acoustic waves (Newell and Aucoin, 1971; L'vov et al., 1997) since in the weakly compressible limit, F-waves tend to be pure acoustic waves. However, the last two mechanisms involving interaction between F-waves and S- or A-waves are completely new. Note finally, that in the weak compressible limit, a similar resonant manifold for Alfvénic waves is obtained for three-wave interactions involving S- and A-waves. Note that a 1D Ansatz (as found in Webb et al., 2000) leaves the FFF resonant manifolds (rays) unchanged, whereas the resonant manifolds of all of the other processes degenerate into points.

It is likely that these different mechanisms of redistribution do not have the same efficiency at large $M_{A}$. (Note that the fast speed of waves does not automatically mean that the nonlinear processes are fast!) To find the relative strength of the different three-wave processes, one has to take into account the competition between the asymptotic decrease of interaction coefficients and the asymptotic amplification since some waves become less dispersive for large $M_{A}$ (the frequency Jacobian becomes large in the collisional integral). The exact answer depends on the asymptotic behaviour in the weakly compressible limit of the collisional integral, which is yet to be derived. At the level of this study, we can only speculate about it and outline different physical possibilities. For example, the most efficient mechanism could be a transfer along spheres. This mechanism does not degenerate Fourier-space by foliating it into noninteracting manifolds and thus, energy can be redistributed throughout $\boldsymbol{k}$-space. Consequently, the study of other sub-redistributions, which could appear later, is unnecessary. However, if the most efficient transfer mechanism degenerates the Fourier-space, such as a transfer along planes or rays, then it is relevant to know the second most efficient mechanism of transfer, because it will tell us how the redistribution takes place at different time scales. For instance, we can imagine a transfer dominated by Alfvénic turbulence, i.e. along planes perpendicular to the $\boldsymbol{B}_{0}$ direction. For this scenario, we see that our knowledge of Alfvénic turbulence (Galtier et al., 2000) could be useful to describe the dynamics at least for small times. On the other hand, if the dominant transfer occurs, similar to pure acoustic waves, i.e. along rays, then the acoustic turbulence developed in Newell and Aucoin (1971) and in L'vov et al. (1997) provides the theory for small times. We see that, in any case, the competition between the different mechanisms gives a richness that we do not have for afvénic turbulence.

An important other question is about the presence (or absence) of double resonances. According to the previous study, 
relevant double resonances are observed for FFF, SSS, SSA and SAA interactions. The presence of double resonances can significantly affect the asymptotic behaviour of certain singular integrals of the form

$\int_{-\infty}^{+\infty} g(\boldsymbol{\kappa}, \boldsymbol{k}) \Delta(h) d \boldsymbol{\kappa}$,

with

$\Delta(h)=\frac{e^{i h t}-1}{i h}$,

whose evaluation is required in obtaining the closure equations (this point is studied in detail in Newell and Aucoin (1971)). Briefly, the reason is that the major contribution to (54) is due to values of $\boldsymbol{\kappa}$ near a zero of $h(\boldsymbol{\kappa} ; \boldsymbol{k})$. If double resonances are present, i.e. if in the Taylor expansion (40) the gradient is zero, then the behaviour of (54) is essentially governed by the next term in the Taylor expansion, which depends on the dimension of the problem. But, as stated previously, such a conclusion depends, of course, on the asymptotic behaviour of interaction coefficients and of the Jacobian in the collisional integral.

\section{Conclusion}

The main result of the present paper is a derivation and a discussion of the fundamental differences between weak Alfvénic turbulence and weak MHD turbulence in the weakly compressible limit, at the level of three-wave interactions. It is shown that the main difference is in the structure of the resonant manifolds and the possible mechanisms of redistribution of spectral densities along the background magnetic field direction $\boldsymbol{B}_{\mathbf{0}}$. However, the relative strength of the different three-wave processes can only be revealed by going further in the calculation. The final goal of such a study is the derivation of a complete weak MHD turbulence theory. This work is currently in progress.

Weak MHD turbulence is a useful theoretical framework to understand media, such as solar wind or the interstellar medium. It gives important information about the effects of a strong magnetised anisotropy on the dynamics of turbulent plasmas. The study in the incompressible case suggests that the energy spectrum of such media, where a moderate anisotropy is measured, must be steeper than the phenomenological IK's prediction (in $k^{-3 / 2}$ ) for isotropic turbulence. Does it mean that the steeper spectrum observed in the solar wind is a signature of the IK spectrum modified by the presence of anisotropy? To answer this important question, it would be interesting to make new analyses of in situ space plasma data where the dependence of the spectral index in the degree of anisotropy would be investigated.

Acknowledgements. Grant from EC (contract FMRX-CT98-0175) is gratefully acknowledged. S. G. would like to thanks J. Büchner and G. Belmont for their invitation to the European Geophysical Society conference hold in Nice in April 2000.

\section{References}

Akhiezer, A., Akhiezer, I. A., Polovin, R. V., Sitenko, A. G., and Stepanov, K. N., Plasma Electrodynamics II : Non-linear Theory and Fluctuations, Pergamon Press, 1975.

Armstrong, J. W., Cordes, J. M., and Rickett, B. J., Density power spectrum in the local interstellar medium, Nature, 291, 561-564, 1981.

Bavassano, B., Dobrowolny, M., Mariani, F., and Ness, N. F., Radial evolution of power spectra of interplanetary Alfvenic turbulence, J. Geophys. Res., 87, 3617-3622, 1982.

Bavassano, B., Dobrowolny, M., Fanfoni, G., Mariani, F., and Ness, N. F., Statistical properties of MHD fluctuations associated with high-speed streams from Helios-2 observations, Solar Phys., 78, 373-384, 1982.

Belcher, J. W. and Davis, L., Large-amplitude Alfvén waves in the interplanetary medium 2, J. Geophys. Res., 76, 3534, 1971.

Benney, D. J. and Newell, A. C., Random wave closure, Studies in Applied Math., 48, 29-53, 1969.

Bhattacharjee, A., Ng, C. S., and Spangler, S. R., Weakly compressible magnetohydrodynamic turbulence in the solar wind and the interstellar medium, Astrophys. J., 494, 409-418, 1998.

Bhattacharjee, A. and Ng, C. S., Random scattering and anisotropic turbulence of shear-Alfvén wave packets, Astrophys. J., will appear, 2000.

Bieber, J. W., Wanner, W., and Matthaeus, W. H., Dominant twodimensional solar wind turbulence with implications for cosmic ray transport, J. Geophys. Res., 101, 2511-2522, 1996.

Burlaga, L. F. and Goldstein, M. L., Radial variations of large-scale magnetohydrodynamic fluctuations in the solar wind, J. Geophys. Res., 89, 6813-6817, 1984.

Burlaga, L. F., Ness, N. F., and McDonald, F. B., Large-scale fluctuations between $13 \mathrm{AU}$ and $25 \mathrm{AU}$ and their effects on cosmic rays, J. Geophys. Res., 92, 13647-13652, 1987.

Falkovich, G. E. and Shafarenko, A. V., Nonstationary wave turbulence, J. Nonlinear Sci., 1, 457-480, 1991.

Galtier, S., Nazarenko, S. V., Newell, A. C., and Pouquet, A., A weak turbulence theory for incompressible MHD, J. Plasma Physics, 63(5), 447-488, 2000.

Gekelman, W. and Pfister, H., Phys. Fluids, 31, 2017, 1988.

Goldreich, P. and Sridhar, S., Toward a theory of interstellar turbulence. II. Strong alfvénic turbulence, Astrophys. J., 438, 763775, 1995.

Goldreich, P. and Sridhar, S., Magnetohydrodynamic turbulence revisited, Astrophys. J., 485, 680-688, 1997.

Goldstein, M. L., Roberts, D. A., Matthaeus, W. H., Magnetohydrodynamic turbulence in cosmic winds, in Cosmic winds and the heliosphere, eds. Jokipii, J. R., Sonett, C. P., and Giampapa, M. S., Univ. Arizona Press, Tucson, 1997.

Ghosh, S. and Goldstein, M. L., Anisotropy in Hall MHD turbulence due to a mean magnetic field, J. Plasma Physics, 57, 129_ 154, 1997.

Grappin, R., Pouquet, A., and Léorat, J., Dependance of MHD turbulence spectra on the velocity field-magnetic field correlation, Astron. Astrophys., 126, 51-58, 1983.

Grappin, R., Mangeney, A., and Marsch, E., On the origin of solar wind turbulence - HELIOS data revisited, J. Geophys. Res., 95, 8197-8209, 1990.

Heiles, C., Goodman, A. A., Mc Kee, C. F., and Zweibel, E. G., Magnetic fields in star-forming regions: observations, in Protostars and Planets IV, Univ. Arizona Press, Tucson, 279, 1993.

Horbury, T., Balogh, A., Forsyth, R. J., and Smith, E. J., Anisotropy 
of inertial range turbulence in the polar heliosphere, Geophys. Rev. Lett., 22, 3405-3408, 1995,

Iroshnikov, P., Turbulence of a conducting fluid in a strong magnetic field, Sov. Astron., 7, 566-571, 1963.

Jeffrey, A. and Taniuti, T., Non-linear wave propagation, Mathematics in science and engineering, 9, Academic Press, New York, 1964.

Kaburaki, O. and Uchida, Y., Magnetohydrodynamic wave-mode coupling. Quantum field-theoretical approach to weakly nonlinear case with application to solar corona heating, Publ. Astron. Soc. Japan, 23, 405-423, 1971.

Kraichnan, R., Inertial-range spectrum of hydromagnetic turbulence, Phys. Fluids, 8, 1385-1387, 1965.

Kinney, R. M. and McWilliams, J. C., Turbulent cascades in anisotropic magnetohydrodynamic, Phys. Rev. E, 57, 71117121, 1998.

Lacaze, R., Lallemand, P., Pomeau, Y., and Rica, S., Dynamical formation of a Bose-Einstein condensate, preprint, 1999.

L'vov, V. S., L'vov, Yu., Newell, A. C., and Zakharov, V., Statistical description of acoustic turbulence, Phys. Rev. E, 56, 390-405, 1997.

Marsch, E. and Mangeney, A., Ideal MHD equations in terms of compressive Elsässer variables, J. Geophys. Res., 92, 73637367, 1987.

Marsch, E. and Tu, C. Y., Dynamics of correlation functions with Elsässer variables for inhomogeneous MHD turbulence, J. Plasma Physics, 41, 479-491, 1989.

Marsch, E. and Tu, C. Y., On the radial evolution of MHD turbulence in the inner heliosphere, J. Geophys. Res., 95, 8211-8229, 1990a.

Marsch, E. and Tu, C. Y., Spectral and spatial evolution of compressible turbulence in the inner solar wind, J. Geophys. Res., 95, 11945-11956, 1990b.

Marsch, E. and Tu, C. Y., Correlations between the fluctuations of pressure, density, temperature and magnetic field in the solar wind, Ann. Geophysicae, 11, 659-677, 1993.

Marsch, E. and Tu, C. Y., Non-gaussian probability distributions of solar wind fluctuations, Ann. Geophysicae, 12, 1127-1138, 1994.

Matthaeus, W. H., Ghosh, S., Oughton, S., and Roberts, D. A., Anisotropic three dimensional MHD turbulence, J. Geophys. Res., 101, 7619-7629, 1996.

McIvor, I., The inertial range of weak magnetohydrodynamic turbulence in the interstellar medium, Mon. Not. Roy. Astron. Soc., 178, 85-99, 1977.

Montgomery, D. and Turner, L., Anisotropic magnetohydrodynamic turbulence in a strong external magnetic field, Phys. Fluids, 24, 825-831, 1981.

Montgomery, D. C. and Matthaeus, W. H., Anisotropic modal energy transfer in interstellar turbulence, Astrophys. J., 447, 706707, 1995.

Nakariakov, V. M., Roberts, B., and Petrukhin, N. S., Nonlinear dynamics of fast magnetoacoustic waves ducted by a smooth plasma inhomogeneity, J. Plasma Physics, 58, 315-327, 1997.

Nazarenko, S. V., Newell, A. C., and Galtier, S, Non-local MHD turbulence, Physica D, in press.

Newell, A. C. and Aucoin, P. J., Semidispersive wave systems, J. Fluid Mech., 49, 593-609, 1971.

$\mathrm{Ng}, \mathrm{C}$. S. and Bhattacharjee, A., Interaction of shear-Alfvén wave packets: implication for weak magnetohydrodynamic turbulence in astrophysical plasmas, Astrophys. J., 465, 845-854, 1996.
$\mathrm{Ng}, \mathrm{C} . \mathrm{S}$. and Bhattacharjee, A., Scaling of anisotropic spectra due to the weak interaction of shear-Alfvén wave packets, Phys. Plasma, 4, 605-610, 1997.

Oughton, S., Priest, E. R., and Matthaeus, W. H., The influence of a mean magnetic field on three-dimensional magnetohydrodynamic turbulence, J. Fluid Mech., 280, 95-117, 1994.

Parker, E. N., Spontaneous current sheets in magnetic fields with applications to stellar X-rays, Oxford University Press, Oxford, 1994.

Pouquet, A., Magnetohydrodynamic turbulence, in Les Houches Summer School on Astrophysical Fluid Dynamics, July 1987, Session XLVII, Eds. J. P. Zahn and J. Zinn-Justin, Elsevier, 139227, 1993.

Priest, E. R., Solar Magnetohydrodynamics, D. Reidel Pub. Comp., 1982.

Roberts, D. A., Ghosh, S., and Goldstein, M. L., Nonlinear evolution of interplanetary Alfvénic fluctuations with convected structures, Geophy. Res. Lett., 23, 591-594, 1996.

Robinson, D. and Rusbridge, M., Structure of turbulence in the zeta plasma, Phys. Fluids, 14, 2499-2511, 1971.

Sagdeev, R. and Galeev, A., Nonlinear Plasma Theory, Benjamin Inc., 1969.

Spangler, S. R. and Gwinn, C. R., Evidence for an inner scale to the density turbulence in the interstellar medium, Astrophys. J., 353, L29-L32, 1990.

Sridhar, S. and Goldreich, P., Toward a theory of interstellar turbulence. I. Weak alfvénic turbulence, Astrophys. J., 432, 612-621, 1994.

Svistunov, B. V., Highly nonequilibrium Bose condensation in a weakly interacting gas, J. Moscow Phys. Soc, 1, 373-390, 1991.

Taylor, J. B., Relaxation and magnetic reconnection in plasmas, Rev. Mod. Phys., 58, 741-763, 1986.

Tu, C.-Y. A solar wind model with the power spectrum of Alfvénic fluctuations, Solar Phys., 109, 149-186, 1987.

Tu, C. Y. and Marsch, E., MHD structures, waves and turbulence in the solar wind: Observations and theories, Space Sci. Rev., 73, 1-210, 1995.

Vázquez-Semadeni, E., Passot, T., and Pouquet, A., A turbulent model for the interstellar medium I: threshold star formation and self-gravity, Astrophys. J., 441, 702-725, 1995.

Vedenov, A. A., Theory of weakly turbulent plasma, in Reviews of plasma physics, ed. M. A. Leontovich, New York, Consultants Bureau 3, 229-276, 1967.

Webb, G. M., Zakharian, A., Brio, M., and Zank, G. P., Wave interactions in magnetohydrodynamics, and cosmic-ray-modified shocks, J. Plasma Physics, 61(2), 295-346, 1999.

Webb, G. M., Zakharian, A., Brio, M., and Zank, G. P., Nonlinear and three-wave resonant interactions in magnetohydrodynamics, J. Plasma Physics, 63(5), 393-445, 2000.

Zakharov, V. E., The Hamiltonian formalism for waves in nonlinear media with dispersion, Izv. Vyssh. Uchebn. Radio., 17, 431-453, Radiophys. and Quantum Electronics, 1974.

Zakharov, V. E., L'vov, V., and Falkovich, G. E., Kolmogorov spectra of turbulence I: wave turbulence, Berlin: Springer, 1992.

Zank, G. P. and Matthaeus, W. H., Nearly incompressible Fluids II: Magnetohydrodynamics, turbulence, and waves, Phys. Fluids A, 5, 257-273, 1993.

Zhou, Ye and Matthaeus, W. H., Non-WKB evolution of solar wind fluctuations - A turbulence modeling approach, Geophy. Res. Lett., 16, 755-758, 1989. 